\title{
Statistical-Mechanics-Inspired Optimization of Sensor Field Configuration for Detection of Mobile Targets
}

\author{
Kushal Mukherjee, Student Member, IEEE, Shalabh Gupta, Member, IEEE,
} Asok Ray, Fellow, IEEE, and Thomas A. Wettergren, Senior Member, IEEE

\begin{abstract}
This paper presents a statistical-mechanics-inspired procedure for optimization of the sensor field configuration to detect mobile targets. The key idea is to capture the lowdimensional behavior of the sensor field configurations across the Pareto front in a multiobjective scenario for optimal sensor deployment, where the nondominated points are concentrated within a small region of the large-dimensional decision space. The sensor distribution is constructed using location-dependent energy-like functions and intensive temperature-like parameters in the sense of statistical mechanics. This low-dimensional representation is shown to permit rapid optimization of the sensor field distribution on a high-fidelity simulation test bed of distributed sensor networks.
\end{abstract}

Index Terms-Gibbs distribution, mobile target detection, optimization of the sensor field configuration, sensor networks.

\section{NOMENCLATURE}

$e^{k}(i) \quad$ Scalar energy corresponding to the $k$ th intensive parameter and the $i$ th state.

$E^{k} \quad$ Energy vector corresponding to the $k$ th intensive parameter.

$f_{S} \quad$ Sensor density function.

$f_{T} \quad$ Distribution of expected targets.

$i \quad$ Index for the state $(i \in\{1,2, \ldots, n\})$.

$J \quad$ Combined objective functional.

$k \quad$ Index for the intensive parameters $(k \in\{1,2, \ldots, K\})$.

$K \quad$ Total number of intensive parameters.

$k_{d} \quad$ Number of sensors confirming target detection.

$n \quad$ Number of states.

$N \quad$ Number of sensors.

Manuscript received April 12, 2010; revised October 18, 2010; accepted November 9, 2010. Date of publication December 17, 2010; date of current version May 18, 2011. This work was supported in part by the U.S. Office of Naval Research under Grant N00014-09-1-0688 and in part by the U.S. Army Research Laboratory and the U.S. Army Research Office under Grant W911NF-07-1-0376. Any opinions, findings, and conclusions or recommendations expressed in this publication are those of the authors and do not necessarily reflect the views of the sponsoring agencies. This paper was recommended by Associate Editor S. Ferrari.

K. Mukherjee, S. Gupta, and A. Ray are with the Department of Mechanical Engineering, Pennsylvania State University, University Park, PA 16802 USA (e-mail: kum162@psu.edu; szg107@psu.edu; axr2@psu.edu).

T. A. Wettergren is with the Naval Undersea Warfare Center, Newport, RI 02841-1708 USA (e-mail: t.a.wettergren@ieee.org).

Color versions of one or more of the figures in this paper are available online at http://ieeexplore.ieee.org.

Digital Object Identifier 10.1109/TSMCB.2010.2092763
$P_{D} \quad$ Probability of detection of a target by a single sensor (target within distance $R_{d}$ from the sensor).

$P_{F} \quad$ Probability of a single sensor false alarm rate.

$P_{F T} \quad$ Probability of a specific false track.

$P_{F S} \quad$ Probability of a false search.

$P_{S T} \quad$ Probability of detection of a specific track.

$P_{S S} \quad$ Probability of a successful search.

$R_{d} \quad$ Radius of detection of a sensor.

$\mathcal{S} \quad$ Surveillance region.

$W_{i} \quad$ Gaussian-mixture weight corresponding to state $i$.

W Weights in vector form.

$\mathbf{W}^{*}(\alpha)$ Optimal weights/distribution for a given $\alpha$.

$x_{i} \quad x$-position of the Gaussian mean for state $i$.

$y_{i} \quad y$-position of the Gaussian mean for state $i$.

$\alpha \quad$ Tradeoff variable between $\log \left(P_{S S}\right)$ and $\log \left(P_{F S}\right)$.

$\beta^{k} \quad k$ th intensive parameter.

$\phi_{\Omega_{T}} \quad$ Probability of finding sensors within the region $\Omega_{T}(x, y, \theta)$.

$\lambda_{i} \quad i$ th largest eigenvalue of $H$.

$\eta \quad$ Threshold for approximation.

$\Omega_{T} \quad$ Pill-shaped region around a target.

$\sigma \quad$ Variance of each component in the Gaussian mixture.

\section{INTRODUCTION}

$\mathbf{R}$ ECENT advances in sensor technology and the development of powerful mobile computational platforms have led to the usage of distributed sensor networks for the tracking of moving targets (e.g., undersea autonomous vehicles and weapon systems) [1]-[3]. Typically, such sensor networks consist of a large number (e.g., $\approx 100-1000$ ) of inexpensive sensor nodes that cover large surveillance regions. As an advantage of the parallel operations, distributed sensor networks have a larger coverage than their conventional counterparts in terms of both area and speed. In addition, distributed sensor networks are robust to random variations in operating conditions and unexpected failures that may happen during the course of operation [2].

Typically, in a sensor field, each sensor is designed with a limited autonomous target detection capability to reduce the sensor cost, and the information from multiple sensors is combined to compensate for the limited coverage provided by the individual sensors. This process of requiring multiple sensor signals for a successful target detection is referred to as track-before-detect [4], since a confident system-level detection decision is not made until a set of multiple sensor detections 
occur in a spatiotemporal pattern that is consistent with the expected target motion. Along this line, Wettergren [5] analyzed the performance of the track-before-detect schemes for the sensor networks. Furthermore, a method that is used to optimize the sensor field configuration for an efficient target detection was proposed using different performance metrics [6].

A particular problem of interest is that of the optimization of the sensor field configuration for the purpose of target track coverage. In contrast to point coverage that requires optimal search solutions in finding stationary objects within the surveillance region, target track coverage uses fixed sensors to search for moving targets in the surveillance region. The sensor density that is required for target track coverage is lower than that for point coverage as the moving targets leave a larger footprint over time, and therefore, they may be detected by multiple sensors. Stone [7] presented a survey of optimal target search techniques, where only the independent noncollaborative sensors were used. Meguerdichian et al. [8] examined the performance of an ad-hoc network to address the problem of sensor allocation to achieve the target tracking capabilities over the region of interest. Ram et al. [9] analyzed the track-coverage property of a random sensor network and provided asymptotic results for its performance. Cloqueur et al. [10] incorporated a deployment cost to choose the number of sensors in a region. Track-coverage assessment of a given configuration was addressed by Baumgartner and Ferrari [11] for the reorganization of the sensor field to achieve the maximum coverage. The trackbefore-detect-based optimal control of sensor reorganization was analyzed by Baumgartner et al. [12]. Recently, the concepts of statistical mechanics [13] have been extended to various fields such as statistical learning [14], communication networks [15], autonomous systems [16], [17], swarm control [18], [19], and optimization [20], [21].

Optimal configurations work well for problems of limited duration. However, for long-term operation, the tradeoff between detection and false alarm performance may change throughout the deployment. In such cases, the reorganization of these fields is important for persistent operation to accommodate changes in the operational intent. The reorganization of the sensor field requires reoptimization to determine its new configuration. However, in computation and communication constrained environments, computationally efficient methods for rapid optimization are required. In this regard, this paper presents a statistical-mechanics-inspired method for the rapid optimization of the sensor field configuration that provides a set point for the new configuration. A major contribution of this paper is the development of an analytical tool to facilitate the rapid multiobjective optimization of the sensor placement for the detection of mobile targets. In this regard, the largedimensional space of the sensor field configurations is expressed as a function of a low-dimensional set of intensive parameters (e.g., temperature, pressure, and chemical potential in statistical mechanics). Consequently, the optimization process becomes rapid because the search for the optimal configuration is performed in a significantly reduced dimensional space. The algorithms for the optimization of the sensor field configuration have been validated on a simulation test bed of distributed sensor networks.



Fig. 1. Detection region $\Omega_{T}(x, y, \theta)$ around a target track originating at $(x, y)$ and heading along a direction $\theta$ for a time interval $\delta t$.

This paper is organized in six sections (including the current section) and an Appendix. Section II describes the detection model that is used to obtain the probabilities of the successful and false search. Section III poses the problem of rapid optimization based on the concepts of statistical mechanics. Section IV formulates the optimal algorithms for the sensor field configuration in the setting of the Gibbs distribution, and it is supported by the Appendix. Pertinent results are presented in Section V. This paper is concluded in Section VI, with recommendations for future research.

\section{Performance Model for Target Detection}

This section describes a model [5] for the target detection performance of a typical distributed sensor network. The global performance is characterized by the following two measures:

1) the probability of a successful search $\left(P_{S S}\right)$, which is defined by the multisensor target detection;

2) the probability of a false search $\left(P_{F S}\right)$, which is defined by the multisensor false alarms.

While the details of computing the aforementioned parameters in a sensor network are reported in the previous publication [5], the underlying model is briefly described here for completeness.

A collection of $N$ homogenous sensors is deployed in a given surveillance region $\mathcal{S}$, where it may be necessary to update the original configuration in real time for the improvement of the detection performance. The sensor field is assumed to have the following characteristics: 1) each sensor has a sensing radius $R_{d}$, within which it may successfully detect a mobile target with a probability $P_{D}$, and 2) each sensor has a specified false alarm rate $\left(P_{F}\right)$, i.e., the probability of false alarm per unit time. The probability of a false search $\left(P_{F S}\right)$ is reduced by the requirement of multiple detections by different sensors, occurring in a sequence that is spatiotemporally consistent with the expected target motion before confirming a target. In accordance with the track-before-detect paradigm [4], a moving target is detected if the $k_{d}$ (typically $k_{d}=3$ or 4 ) sensors detect the target within a specified time interval $\delta t$ and a spatial region, as shown in Fig. 1. This time interval $\delta t$ is chosen such that the expected target velocity is approximately constant over the interval. Such an approximation is consistent with the Markov modeling assumptions of the moving targets that are commonly used in the target tracking community [22]. 
Fig. 1 shows a target track originating at location $(x, y)$ and moving at a heading $\theta$ over a time interval $\delta t$. The region $\Omega_{T}(x, y, \theta)$ around this target track corresponds to the region of target detectability over time interval $\delta t$. This region represents a subset of the space in which the sensors have an opportunity to detect the specified target track within the sensing radius $R_{d}$.

Let the sensor distribution be denoted with $f_{S}(x, y)$. Then, the probability of finding a sensor in a region $\Omega_{T}$ is given by

$$
\phi_{\Omega_{T}}=\int_{\left(x^{\prime}, y^{\prime}\right) \in \Omega_{T}} f_{S}\left(x^{\prime}, y^{\prime}\right) d x^{\prime} d y^{\prime} .
$$

Furthermore, the probability of a single sensor detecting the target is given by $P_{D} \phi_{\Omega_{T}}$. For a successful target detection, it is required that at least $k_{d}$ out of $N$ sensors independently detects the target within the region $\Omega_{T}$ (each with equal probability $P_{D} \phi_{\Omega_{T}}$ ). This is modeled by the Poisson distribution [2], [5] for a large numbers of sensors $N$ and is given as

$$
P_{S T}\left(k_{d}, \Omega_{T}\right)=1-\exp \left(-N P_{D} \phi_{\Omega_{T}}\right) \sum_{m=0}^{k_{d}-1} \frac{\left(N P_{D} \phi_{\Omega_{T}}\right)^{m}}{m !}
$$

where $P_{S T}\left(k_{d}, \Omega_{T}\right)$ is the probability of detecting a single target that moved along the target path in the region $\Omega_{T}$.

Let the target track distribution be denoted by a joint probability density function $f_{T}(x, y, \theta)$, i.e., the likelihood of a target being present at location $(x, y)$, with heading $\theta$. Thus, the overall probability of a successful search $\left(P_{S S}\right)$ that is relative to this distribution of targets is given by

$$
P_{S S}\left(k_{d}\right)=\int_{0}^{2 \pi} \int_{x, y \in \mathcal{S}} P_{S T}\left(k_{d}, \Omega_{T}\right) f_{T} d x d y d \theta .
$$

Along similar lines, the probability of a false search $\left(P_{F S}\right)$ is defined as the probability that at least $k_{d}$ sensor false alarms occur, which are kinematically consistent with a potential target motion. The probability of a single sensor raising a false alarm in a time interval $\delta t$ is $P_{F} \delta t$ for the small values of the false alarm rate $P_{F}$. Similar to (2), the probability of a specific false track in the region $\Omega_{T}$, denoted as $P_{F T}\left(k_{d}, \Omega_{T}\right)$, is given by

$$
\begin{aligned}
P_{F T}\left(k_{d}, \Omega_{T}\right)=1-\exp \left(-N P_{F} \delta t \phi_{\Omega_{T}}\right) & \\
& \times \sum_{m=0}^{k_{d}-1} \frac{\left(N P_{F} \delta t \phi_{\Omega_{T}}\right)^{m}}{m !} .
\end{aligned}
$$

The probabilities of false track events, which are independent for the nonoverlapping regions $\Omega_{T}$, are assumed to be Poisson. Thus, the probability of zero false tracks is given by

$$
\operatorname{Pr}\{0\}=\exp \left(-\frac{1}{A_{\Omega_{T}}} \int_{0}^{\pi} \int_{x, y \in \mathcal{S}} P_{F T}\left(k_{d}, \Omega_{T}\right) d x d y d \theta\right)
$$

where $A_{\Omega_{T}}$ is the area of the region $\Omega_{T}$. Thus, the probability of a false search $P_{F S}$ is given by

$$
P_{F S}\left(k_{d}\right)=1-\operatorname{Pr}\{0\} .
$$



Fig. 2. Partition of the surveillance region and the associated parameters.

The exact procedure for the evaluation of the performance measures, i.e., the probability of a successful search $\left(P_{S S}\right)$ and the probability of a false search $\left(P_{F S}\right)$, is given in [5]. The procedure for determining the optimum configuration of the sensor field for detecting the moving targets is described in [6].

\section{Optimization Problem for Sensor Placement}

As described in the previous section, the performance of a sensor field is measured in terms of the probability of the detection of the moving targets and the associated probability of false alarms. The false alarm objective is included to facilitate the avoidance of sensor clumping, which may cause confusion in a track-before-detect construct. Additional parameters such as tracking accuracy and detection time may also be used in the objective functional. The optimization of the sensor distribution using multiple conflicting objectives leads to a Pareto optimal set, also known as the nondominated set [23] of the sensor field configurations. ${ }^{1}$

Based on the aforementioned performance measures [see (3) and (6)], the objective is to construct a set of nondominated sensor distributions $f_{S}(x, y)$. In this regard, these distributions are modeled as a mixture of multivariate Gaussian density functions, given as

$$
f_{S}(x, y)=\frac{1}{2 \pi \sigma^{2}} \sum_{i=1}^{n} W_{i} \exp \left[-\frac{\left(\left(x-x_{i}\right)^{2}+\left(y-y_{i}\right)^{2}\right)}{2 \sigma^{2}}\right]
$$

where $n$ is the number of components in the Gaussian mixture and $W_{1}, W_{2}, \ldots, W_{n}$ are the mixture weights. The Gaussian mixture centers $\left(x_{i}, y_{i}\right)$ are chosen a priori to lie on a square grid on the search space $\mathcal{S}$ consisting of $n$ cells, as shown in Fig. 2. Each grid cell " $i$ " is assigned a weight $W_{i}$. The parameter $\sigma$ is chosen by taking into account the grid spacing [6]. The weights are normalized to yield a probability mass function, i.e., $\sum_{i=1}^{n} W_{i}=1$. The sensor distribution $f_{S}(x, y)$ can be obtained by computing the weight vector $\mathbf{W}=$ $\left[W_{1}, W_{2}, \ldots, W_{n}\right]$. Therefore, a point in the Pareto set represents a particular choice of the weight vector $\mathbf{W}$ as used in (7).

\footnotetext{
${ }^{1}$ The configurations in the Pareto optimal set are nondominated in the sense that there is no configuration which is an improvement in every objective over any Pareto configuration.
} 
Usually, the Pareto set is a very small subset of achievable configurations. In other words, a vast majority of the sensor field configurations belong to the dominated set. The total number of possible configurations exponentially increases with the number of sensors, implying that the Pareto optimal set forms a small fraction of the entire set of configurations. As a result, most of the current Pareto optimization techniques [24] that utilize the concepts of genetic algorithms or nonlinear programming [23] to optimize the sensor field configuration may take a long time to search the entire configuration space and may still yield an approximate Pareto set. For example, traditional normal boundary intersection (NBI)-based genetic algorithms, such as the genetic-algorithm-based NBI (GANBI), ${ }^{2}$ [24] may be used to obtain a set of sensor distributions that represent a suboptimal Pareto set.

This paper utilizes the solutions obtained from GANBI to create a model of the Pareto set that is dependent on a small number of parameters. Consequently, the reduced dimension of the search space enables rapid optimization using nonlinear programming algorithms (e.g., sequential quadratic programming (SQP) [23]) to obtain a close-to-optimal Pareto set which is likely to be superior to that obtained from GANBI.

To define the objective space, this paper makes use of two competing performance measures: 1) the probability of a successful search $P_{S S}(\mathbf{W})$ and 2) the probability of a false search $P_{F S}(\mathbf{W})$, where $\mathbf{W}$ denotes the weight vector that parameterizes the sensor distribution $f_{S}(x, y)$ [see (7)]. The other parameters, such as $k_{d}, N$, and $f_{T}(x, y, \theta)$, are assumed to be implicitly present in the performance measures. Assuming convexity, the Pareto front is parameterized by constructing a maximization objective function $J$ that is given by the following weighted sum:

$$
J(\mathbf{W}, \alpha)=\alpha \log \left[P_{S S}(\mathbf{W})\right]+(1-\alpha)\left(-\log \left[P_{F S}(\mathbf{W})\right]\right)
$$

where $\alpha \in[0,1]$, i.e., the Pareto front is generated by varying $\alpha$ from zero to one. The Pareto front is a (generally convex) curve in the $\log \left(P_{S S}\right)$ versus $\log \left(P_{F S}\right)$ space. In this space, for a point on the Pareto front with slope $m$, the corresponding $\alpha=$ $(1 / 1+m)$. An optimal sensor field configuration is chosen from the Pareto set by assigning different weights to different performance objectives [i.e., $\alpha$ in (8)]. Formally, the optimal sensor distribution for a given value of $\alpha$ is given by

$$
\mathbf{W}^{*}(\alpha)=\arg \max _{\mathbf{W}} J(\mathbf{W}, \alpha) .
$$

In real-time operations, a supervisor may change the relative weights of the individual objectives depending on the situation, thereby selecting a different point on the Pareto set. Consequently, a different sensor field configuration is generated.

Remark 3.1: The objective function $J$ in (8) may be augmented with additional performance measures, such as tracking

\footnotetext{
${ }^{2} \mathrm{GANBI}$ produces a set of nondominated solutions in the multiobjective space as an estimate of the Pareto front.
}

accuracy and detection time. However, with more than two objective functions, $\alpha$ will become a vector instead of being a scalar. Correspondingly, the dimension of the vector $\alpha$ will be one less than the number of individual objectives, resulting in a Pareto hypersurface. The rest of the analysis described in this paper remains unchanged.

This paper utilizes the concepts of statistical mechanics in constructing a reduced-order model of the Pareto set with a small number of parameters. This model provides a more tractable structure for rapid optimization using nonlinear programming methods. Therefore, in addition to generating a close-to-optimal Pareto front, this approach enables rapid estimation of the parameters for sensor field configuration whenever the relative weight $\alpha$ of the objectives is altered.

The underlying principle of statistical mechanics involves the construction of the energy states, where the equilibrium probability distribution is estimated by maximizing the entropy of the system for a given macroscopic parameter (e.g., energy) [13]. The distribution of the population among various energy states, called as the generalized canonical distribution or the Gibbs distribution [13], is given as

$$
P_{i}=\frac{\exp [-\beta e(i)]}{\sum_{j=1}^{n} \exp [-\beta e(j)]}, \quad i \in\{1,2, \ldots, n\}
$$

where $e(i)$ is an extensive parameter that represents the energy of a particle in state $i$ and $\beta$ is an intensive parameter that represents the inverse temperature. In general, a system is characterized by multiple intensive parameters [13]. In that case, (10) is modified as

$$
P_{i}=\frac{\exp \left[-\sum_{k=1}^{K} \beta^{k} e^{k}(i)\right]}{\sum_{j=1}^{n} \exp \left[-\sum_{k=1}^{K} \beta^{k} e^{k}(j)\right]}, \quad \in\{1,2, \ldots, n\}
$$

where $\beta^{k}$, with $k \in\{1,2, \ldots, K\}$, is the intensive parameters and $e^{k}(i)$, with $k \in\{1,2, \ldots, K\}$, is the extensive parameters that represent the energies of the state $i$.

In this paper, the Gibbs distribution is used to construct a model structure for the rapid optimization of the sensor field configurations. The optimization procedure consists of two phases: 1) (offline) training and 2) (online) operation. For a sensor field, the search space $\mathcal{S}$ is partitioned into $n$ cells to form a grid, and each grid cell is defined as a state, as shown in Fig. 2. In the training phase, the $K$ energy parameters $e^{k}(i)$, with $k \in\{1,2, \ldots, K\}$, are computed for each state $i$ (see Section IV for details). Once computed, the energy parameters are fixed for each state and are kept invariant in the operation phase. The intensive parameters $\beta^{k}$, with $k \in\{1,2, \ldots, K\}$, do not depend on the state $i$. However, they may vary in the operation phase. In the operation phase, moving to a different operating point on the Pareto front corresponds to a change in the intensive parameters while keeping the associated energies $e^{k}(i)$ invariant. Since the intensive parameters are common to all states, they serve as the control parameters for the optimization of the sensor field configuration, which may be broadcasted to the entire network for online operation [25]. 


\section{Gibbs Distribution for Optimizing the SENSOR FIELD CONFIGURATION}

This section describes a method that is used to model the changes in the sensor distribution as the parameter $\alpha$ in (8) varies in the interval $[0,1]$. It is desirable to construct this model of the Pareto set with as few parameters as possible in order to facilitate the real-time adaptation for the sensor field configuration. Any point on the convex Pareto front can be represented as an optimal solution that maximizes $J(\mathbf{W}, \alpha)$ in (8) for some $\alpha \in[0,1]$. Therefore, a continuous one-to-one mapping exists between the Pareto front and the set $[0,1]$. As described in (7), the sensor distribution $f_{S}(x, y)$ is parameterized by the weights $W_{1}, W_{2}, \ldots, W_{n}$ given to the $n$ components in the Gaussian mixture. Furthermore, each point on the Pareto front corresponds to a certain weight vector expressed as $\mathbf{W}^{*}(\alpha)=\left[\begin{array}{llll}W_{1}^{*}(\alpha) & W_{2}^{*}(\alpha) & \cdots & W_{n}^{*}(\alpha)\end{array}\right]$.

\section{A. Model Construction}

The individual weights are modeled by the Gibbs distribution as

$$
W_{i}^{*}(\alpha) \triangleq \exp \left(-\sum_{k=1}^{K} \beta^{k}(\alpha) e^{k}(i)-A(\alpha)\right)
$$

where the energy functions $e^{k}(i)$, with $k \in\{1, \ldots, K\}$ and $K \leq n$, are defined on states $i \in\{1, \ldots, n\}$ and $A(\alpha)$ is the normalizing factor. Note that the intensive parameters $\beta^{k}$, with $k \in\{1, \ldots, K\}$, depend on $\alpha$ but not on the state $i$, while the extensive parameters $e^{k}$, with $k \in\{1, \ldots, K\}$, depend on the state $i$ but not on $\alpha$. Taking the logarithm on both sides and multiplying with the negative one, (12) yields

$$
G_{i}(\alpha) \triangleq-\log \left(W_{i}^{*}(\alpha)\right)=\sum_{k=1}^{K} \beta^{k}(\alpha) e^{k}(i)+A(\alpha)
$$

where $\beta^{k}(\alpha)$ and $A(\alpha)$ depend on the parameter $\alpha$. The term $A(\alpha)$ that is chosen to normalize the distribution is analogous to the free energy in the thermodynamic sense [13].

As stated earlier, the state-dependent $e^{k}(i)$ 's are invariant along the Pareto front, while $\beta^{k}$,s are invariant across all states. The next task is to estimate the values of the statedependent $e^{k}$ 's in the sense of minimum variance. Since these functions are defined over a discrete and finite domain, they can be represented by the corresponding energy vectors: $E^{k} \triangleq$ $\left[\begin{array}{llll}e^{k}(1) & e^{k}(2) & \cdots & e^{k}(n)\end{array}\right]^{T} \forall k \in\{1, \ldots, K\}$. The following constraints are imposed without loss of generality.

1) The Euclidean norm of the energy vector is set as $\left\|E^{k}\right\|_{2}=1 \forall k \in\{1, \ldots, K\}$. The scaling factor of the energy vector is absorbed into $\beta^{k}$.

2) Any constant added to the energy vectors is absorbed into the bias function $A(\alpha)$, and it does not affect the distribution. Hence, the sum of the elements of the energy vector is constrained to be zero by subtracting the mean of the energy from each element. Thus

$$
\sum_{i=1}^{n} e^{k}(i)=0 \quad \forall k \in\{1, \ldots, K\} .
$$

3) Given $K$ linearly independent energy vectors $E^{1}, E^{2}$, $\ldots, E^{K}$, it is possible to construct an orthogonal set of $K$ vectors that span the same space. In this set of basis vectors, the coefficients $\beta^{k}(\alpha)$ are calculated by an appropriate transformation. Therefore, it is assumed that the energy vectors are mutually orthogonal. Thus

$$
\left(E^{k_{1}}\right)^{T}\left(E^{k_{2}}\right)=\delta_{k_{1} k_{2}} \quad k_{1}, k_{2} \in\{1, \ldots, K\}
$$

where $\delta_{k_{1} k_{2}}$ is the Kronecker delta function.

Upon imposing the aforementioned constraints and summing (13) over all $n$ states, it follows that

$$
\begin{aligned}
\sum_{i=1}^{n} G_{i}(\alpha) & =\sum_{k=1}^{K} \beta^{k}(\alpha) \sum_{i=1}^{n} e^{k}(i)+\sum_{i=1}^{n} A(\alpha) \\
& \Rightarrow A(\alpha)=\frac{1}{n} \sum_{i=1}^{n} G_{i}(\alpha) \quad \text { by }(14) .
\end{aligned}
$$

By setting $\widetilde{G}_{i}(\alpha) \triangleq G_{i}(\alpha)-A(\alpha)$, a vector is defined as

$$
\widetilde{\mathbf{G}}(\alpha) \triangleq\left[\begin{array}{llll}
\widetilde{G}_{1}(\alpha) & \widetilde{G}_{2}(\alpha) & \cdots & \widetilde{G}_{n}(\alpha)
\end{array}\right]^{T} .
$$

The components of vector $\widetilde{\mathbf{G}}(\alpha)$ also sum up to zero, i.e., the vectors $\widetilde{\mathbf{G}}(\alpha)$ and $E^{1}, E^{2}, \ldots, E^{K}$ lie in the same $(n-1)$ dimensional subspace of $\mathbb{R}^{n}$.

\section{B. Offline Estimation of the Energy Vectors}

In the training phase, the energy vectors are estimated from a collection of points in the Pareto set to represent the nondominated sensor field distributions. In this paper, these points are generated by a genetic-algorithm-based multiobjective optimization algorithm (GANBI) [24].

Let the training data set consist of $L$ samples, and let the $n$-dimensional weight vectors that correspond to these sensor distributions be given by $\mathbf{W}^{1}, \mathbf{W}^{2}, \ldots, \mathbf{W}^{L}$. For a reliable statistical analysis, $L$ must be sufficiently larger than the number of states $n$. Let $\mathbf{G}^{l}$ be a transformation of the weight vector $\mathbf{W}^{l}$ such that

$$
\mathbf{G}^{l} \triangleq-\log \left(\mathbf{W}^{l}\right), \quad l \in\{1, \ldots, L\} .
$$

As defined earlier, the vector $\widetilde{\mathbf{G}}^{l}$ is obtained as

$$
\widetilde{\mathbf{G}}^{l} \triangleq \mathbf{G}^{l}-\left(\frac{1}{n} \sum_{i=1}^{n} G_{i}^{l}\right)[1,1, \ldots, 1]_{n \times 1}^{T}, \quad l \in\{1, \ldots, L\} .
$$

The orthonormal vectors $E^{1}, E^{2}, \ldots, E^{K}$ are estimated by minimizing the sum of the squared distances between the vectors $\widetilde{\mathrm{G}}^{l}$ and their projections onto the space spanned by $E^{1}, E^{2}, \ldots, E^{K}$. To this end, a cost functional is defined as

$$
\mathfrak{L}=\sum_{l=1}^{L}\left\|\widetilde{\mathbf{G}}^{l}-\sum_{k=1}^{K} E^{k}\left(\left(E^{k}\right)^{T} \widetilde{\mathbf{G}}^{l}\right)\right\|^{2}
$$

where $\mathfrak{L}$ is minimized by obtaining its vector derivatives with respect to the energy vectors $E^{1}, E^{2}, \ldots, E^{K}$ and by setting 
them to be equal to zero. The orthonormality constraints are incorporated by means of Lagrange multipliers (see the Appendix for details). The energy vectors are obtained as the eigenvectors of the self-adjoint and positive semidefinite matrix $H=$ $\left(\sum_{l=1}^{L}\left(\widetilde{\mathbf{G}}^{l}\right)\left(\widetilde{\mathbf{G}}^{l}\right)^{T}\right)$ such that

$$
H E=\lambda E .
$$

The vectors $E^{1}, E^{2}, \ldots, E^{K}$ obtained by the discrete Karhunen-Loéve expansion [26] are real and mutually orthogonal. The eigenvalues are ordered as $\lambda_{1} \geq \lambda_{2} \geq \cdots \geq \lambda_{n} \geq 0$. The eigenvectors corresponding to the largest $K$ eigenvalues, where $K \leq n$, are chosen such that the cumulative sum $(\eta)$ of the remaining $(n-K)$ eigenvalues is bounded above by a positive threshold $\eta_{\tau} \ll 1$

$$
\eta \triangleq \frac{\sum_{i=K+1}^{n} \lambda_{i}}{\sum_{i=1}^{n} \lambda_{i}} \leq \eta_{\tau}
$$

As described earlier, the energy vectors corresponding to each state form the local potentials, and they are identified a priori in the (offline) training phase. The training data set of the nondominated sensor distributions described by the weight vectors $\mathbf{W}^{l}(l=1, \ldots, L)$ are obtained using the genetic algorithms. This data set is suboptimal compared to the ideal Pareto front. Although the values of $\beta^{k}$, with $k=1, \ldots, K$, may be found for each sensor distribution in the training set, the weight vectors $\mathbf{W}^{l}(l=1, \ldots, L)$ are not labeled with the corresponding values of $\alpha$, making it impossible to obtain $\beta^{k}$ as a function of $\alpha$. This issue is addressed in the (online) operation phase.

\section{Online Operation}

The probability of a sensor occupying a state $i$ is governed by a combination of global parameters $\beta^{k}$ 's and local energy parameters $e^{k}(i)$ 's. Given the energy vectors, as obtained in the training phase, the weight vector $\mathbf{W}$ depends on the values of the intensive parameters $\beta^{k}$, with $k=1, \ldots, K$, and it is given by

$$
W_{i}=\frac{\exp \left(-\sum_{k=1}^{K} \beta^{k} e^{k}(i)\right)}{\sum_{j=1}^{n} \exp \left(-\sum_{k=1}^{K} \beta^{k} e^{k}(j)\right)}, \quad i=1, \ldots, n
$$

where $K$ is much smaller than the number of states $n$. For a given value of $\alpha$, the objective functional $J(\alpha)$ [see (8)] is constructed as a linear combination of the probabilities of the successful and false searches. The aim here is to find the weight vector $\mathbf{W}(\alpha)$ that maximizes the objective $J(\alpha)$. However, the weight vectors are functions of $\beta^{k}$, with $k=1, \ldots, K$, that belong to an abstract $K$-dimensional space. The task of finding the optimal weight vector $\mathbf{W}(\alpha)$ is reduced to that of finding the optimal parameters $\beta^{k}(\alpha)$, which maximize $J(\alpha)$. Due to the small dimensionality of the search space (i.e., the space of $\beta^{k}$ 's), the SQP algorithm is suitable in computing $\beta^{k}(\alpha)$. Thus, the supervisory controller may choose an operating point in the $K$-dimensional space of $\beta^{k}$ 's to achieve the global objectives for a given $\alpha$.

\section{Results AND Discussion}

This section presents the results generated by using the multiple-intensive parameter Gibbs distribution to model the Pareto front of the nondominated sensor field configurations. The competing performance criteria in the Pareto front are the probability of a successful search $\left(P_{S S}\right)$ and the probability of a false search $\left(P_{F S}\right)$ for the moving targets. While the details are reported in [6], a brief description for the evaluation of $P_{S S}$ and $P_{F S}$ for a given sensor field configuration has been described in Section II. In the test problem, the surveillance region is divided into a $20 \times 20$ square lattice to yield $n=400$ grid cells. The sensor field configuration, expressed as a probability density, is modeled by a Gaussian mixture centered at each of the grid points [see (7)]. The distribution of the sensors is completely parameterized by a 400 -element weight vector (i.e., $n=400$ ), resulting in 399 independent parameters because of the constraint $\sum_{i=1}^{400} W_{i}=1$. The global performance parameters $P_{S S}$ and $P_{F S}$ have been evaluated based on the following: 1) a uniform distribution of the target tracks, i.e., $f_{T}(x, y, \theta)=\left(1 / 2 \pi L^{2}\right)$, and 2) a skewed distribution of the target tracks $f_{T}(x, y, \theta)=\left(1+\cos (\theta) / 2 \pi L^{2}\right)$ in the domain of the $L \times L$ surveillance region. In these simulation scenarios, the total number of sensors required to be placed is chosen to be $N=60$, with the cumulative point coverage of all the sensors amounting to only $9 \%$ of the surveillance region. In accordance with the track-before-detect strategy, the number of sensors $k_{d}$ that must detect a target to confirm its presence is chosen as two for the results that follow.

A GANBI code [24] has been adopted to solve the multiobjective optimization problem and to obtain the approximate Pareto front of sensor distributions, where $8 \mathrm{~b}$ are used to represent each weight $W_{i}$ in the genetic program [6]. With a population size of 100 , the genetic algorithm has been run for 300 generations to obtain a good spread of points along the Pareto front, where each point corresponds to a set of 400 weights that are used to describe the sensor distribution. As with any genetic-algorithm-based approach, the generated Pareto front is always approximate.

The algorithm presented in this paper extracts the lowdimensional characteristics of the approximate Pareto front in the space of the weights $W_{i}$ and formulates a tractable optimization problem with fewer parameters in order to refine and improve the Pareto front. To this end, the energy vectors $E^{1}, E^{2}, \ldots, E^{K}$ are obtained from the set of approximate Pareto optimal sensor distributions as obtained from GANBI [24]. The number of energy vectors $K$ is given by (22) after an appropriate choice of threshold $\eta_{T}$.

Once the energy vectors are evaluated, the sensor field distribution, given by the weights $W_{i}$, is parameterized in terms of the intensive parameters $\beta^{1}, \beta^{2}, \ldots, \beta^{K}$ [see (23)]. Given an objective function in terms of $\alpha$ [see (8)] and keeping the energy vectors invariant, the optimal sensor distribution parameters $\beta^{1}, \beta^{2}, \ldots, \beta^{K}$ are obtained by using the SQP algorithm as follows:

$$
\left(\beta^{1}(\alpha), \ldots, \beta^{K}(\alpha)\right)^{*}=\arg \max _{\beta^{1}, \ldots, \beta^{K}} J\left(\mathbf{W}\left(\beta^{1}, \ldots, \beta^{K}\right), \alpha\right) .
$$




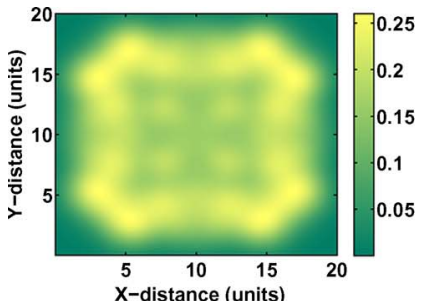

(a)

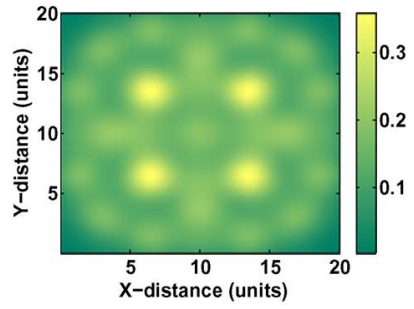

(c)

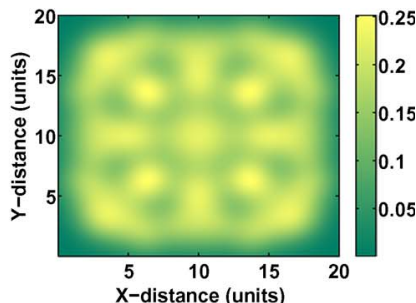

(b)



(d)

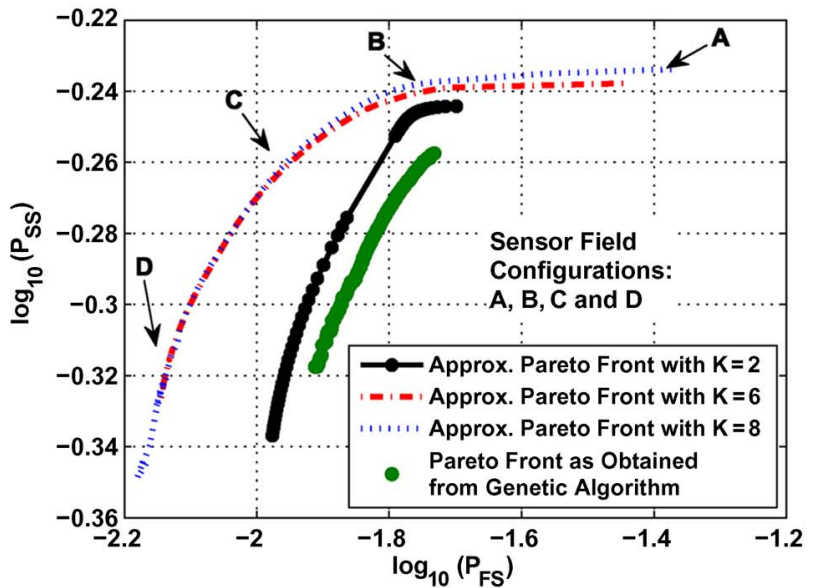

(e)

Fig. 3. Sensor density per unit area corresponding to the points on the Pareto front $(K=8)$ for the uniform target distribution $f_{T}(x, y, \theta)=\left(1 / 2 \pi L^{2}\right)$. (a) Configuration A: $P_{S S}=0.584$ and $P_{F S}=0.043$. (b) Configuration B: $P_{S S}=0.577$ and $P_{F S}=0.017$. (c) Configuration C: $P_{S S}=0.549$ and $P_{F S}=0.011$. (d) Configuration D: $P_{S S}=0.485$ and $P_{F S}=0.007$. (e) Pareto fronts with two, six, and eight intensive parameters.

A complete Pareto front is generated by changing the scalar $\alpha$ in the objective function [in (8)] over a desired range to obtain the optimal values of the parameters $\beta^{1}, \beta^{2}, \ldots, \beta^{K}$. The bottom plate in Fig. 3 shows the estimated Pareto fronts with increasing numbers $(K)$ of intensive parameters for the uniform target distribution $f_{T}(x, y, \theta)=\left(1 / 2 \pi L^{2}\right)$. Thus, the 399 -dimensional space of the weight vectors is reduced to a $K$-dimensional space of intensive parameters. The sensor densities per unit area displayed in the four plates in Fig. 3(a)-(d) correspond to the four points marked as A, B, C, and D on the Pareto front in Fig. 3(e), respectively. Similar results are shown in Fig. 4 for a skewed target distribution $f_{T}(x, y, \theta)=$ $\left(1+\cos (\theta) / 2 \pi L^{2}\right)$. Although the Pareto fronts become more accurate with a larger $K$, the incremental improvements do not justify the increased computational complexity beyond a certain point (in this case, $K=6$ ). The errors due to the reduction in the order of the decision space decrease with an increase in the number of intensive parameters, as shown in the plots in Fig. 5, and they are given by $\eta$ in (22). The approximate computation

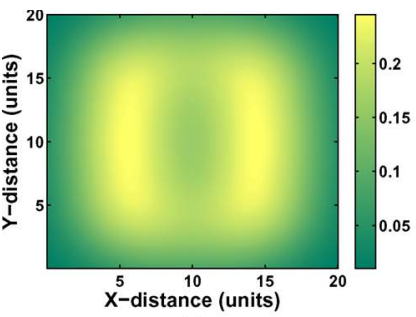

(a)

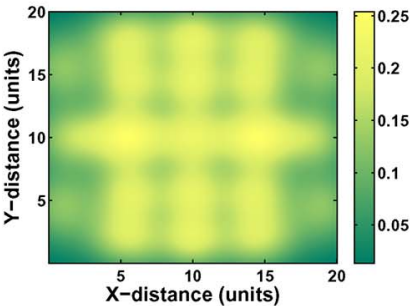

(c)

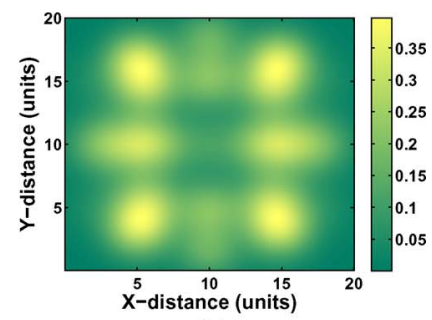

(b)

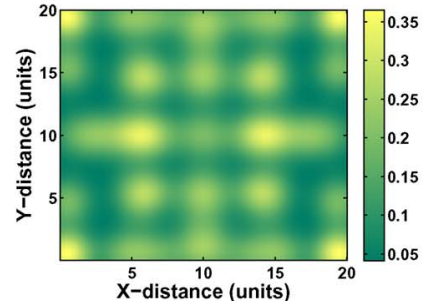

(d)



(e)

Fig. 4. Sensor density per unit area corresponding to the points on the Pareto front $(K=7)$ for the skewed target distribution $f_{T}(x, y, \theta)=$ $\left(1+\cos (\theta) / 2 \pi L^{2}\right)$. (a) Configuration A: $P_{S S}=0.581$ and $P_{F S}=0.035$ (b) Configuration B: $P_{S S}=0.575$ and $P_{F S}=0.023$. (c) Configuration C: $P_{S S}=0.544$ and $P_{F S}=0.016$. (d) Configuration D: $P_{S S}=0.459$ and $P_{F S}=0.010$. (e) Pareto fronts with three and seven intensive parameters.

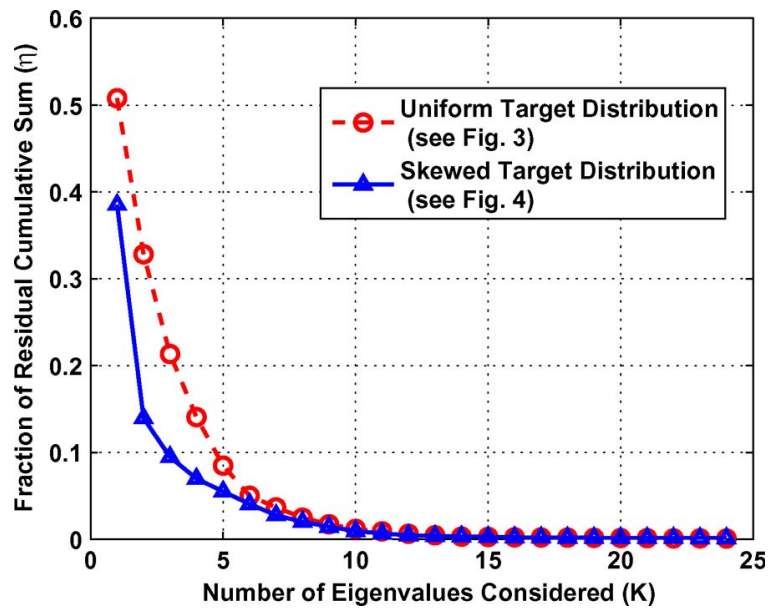

Fig. 5. Error due to a reduced-order decision space.

times for the algorithms are shown in Table I. For the example described in Fig. 3, the genetic algorithm (GANBI) used to obtain the initial Pareto front takes $\sim 6100 \mathrm{~s}$, and it is usually 
TABLE I

COMPARISON OF THE COMPUTATION TIME FOR THE Algorithms

\begin{tabular}{lllll}
\hline & \multirow{2}{*}{ GANBI } & \multicolumn{3}{c}{ Statistical Mechanics-inspired } \\
& & $\mathrm{Kapid}$ & $\begin{array}{c}\text { Optimization } \\
\text { Method }\end{array}$ \\
& & $\mathrm{K}=6$ & $\mathrm{~K}=6$ & $\mathrm{~K}=8$ \\
\hline \hline $\begin{array}{l}\text { Optimization } \\
\text { for a given } \alpha\end{array}$ & N.A. ${ }^{a}$ & $3 \mathrm{~s}$ & $20 \mathrm{~s}$ & $30 \mathrm{~s}$ \\
& & & & \\
$\begin{array}{l}\text { Optimization } \\
\text { for Pareto front }\end{array}$ & $6100 \mathrm{~s}$ & $60 \mathrm{~s}^{b}$ & $400 \mathrm{~s}^{b}$ & $600 \mathrm{~s}^{b}$ \\
\hline
\end{tabular}

executed offline. Once the energy vectors are obtained, the procedure for optimizing the parameters $\beta^{1}, \beta^{2}, \ldots, \beta^{K}$ is relatively less time consuming. Given a value of $\alpha$, this optimization procedure takes 2,20 , and 30 s for $K=2,6$, and 8 , respectively. These results support the applicability of the proposed method for the purpose of the real-time optimization of the sensor placement for target detection.

Remark 5.1: The computation time for the execution of the GANBI strongly depends on the number of states $n$. A larger $n$ may lead to a greater accuracy in determining the sensor configuration at the cost of an increased computational load (i.e., execution time and memory requirements). On the other hand, the complexity of the online statistical-mechanics-inspired method depends on the number of intensive parameters $K$ that can be chosen based on the information provided in Table I.

The sensor distributions in Figs. 3(a) and 4(a) correspond to the case where the maximum weight is given to the probability of a successful search (i.e., $\alpha=1$ ). Fig. 3(a) considers the targets that may move in any direction with equal probability. As a result, the sensor distribution is circularly symmetric and is in the shape of a ring. On the other hand, Fig. 4(a) considers the target movements predominantly from left to right. Consequently, the sensor distribution is in the form of barriers spanning from top to bottom. In both the cases, as the weight on the probability of a successful search is reduced ( $\alpha$ is reduced), the sensor distribution distorts are a manner to effectively reduce the probability of a false search.

\section{SUMmARY AND CONCLUSION}

This paper has presented a methodology for the rapid optimization of the sensor field configuration for a collaborative network for the detection of the moving targets. The underlying algorithms are based on the concepts of equilibrium statistical mechanics, where the Gibbs distribution has been used to model the spatial sensor distribution. In particular, the number of intensive parameters is significantly smaller than the number of states. The energy-like quantities are spatially dependent, but they are independent from the operating point on the Pareto front. On the other hand, the intensive parameters are spatially invariant, but they are dependent on the location of the operating point on the Pareto front. This approach to suboptimal representation attempts to capture the low-dimensional behavior of the nondominated configurations of a very high dimensional system. The following topics are under active research.

1) The sensitivity analysis of the sensor distribution with respect to the intensive parameters and its effects on the computation time for optimization.
2) The extension of the Gibbs distribution to the generalized exponential distributions for modeling the Pareto front.

3) The analysis of the computational complexity versus the accuracy tradeoff in the optimization process by choosing different numbers of intensive parameters.

4) The reorganization of the sensor fields in real time to detect the mobile targets and the analysis of the actual sensor trajectories during the transition. The reorganization may also be addressed by sensor activation and deactivation in various parts of the field.

\section{APPENDIX \\ ESTIMATION OF THE ENERGY VECTORS}

This Appendix outlines a procedure for estimating the energy vectors $E^{k}$ 's by minimizing the cost function $\mathfrak{L}$ defined in (20)

$$
\begin{aligned}
\mathfrak{L}= & \sum_{l=1}^{L}\left(\widetilde{\mathbf{G}}^{l}-\sum_{k=1}^{K} E^{k}\left(\left(E^{k}\right)^{T} \widetilde{\mathbf{G}}^{l}\right)\right)^{T} \\
& \times\left(\widetilde{\mathbf{G}}^{l}-\sum_{k=1}^{K} E^{k}\left(\left(E^{k}\right)^{T} \widetilde{\mathbf{G}}^{l}\right)\right) \\
= & \sum_{l=1}^{L}\left(\widetilde{\mathbf{G}}^{l}\right)^{T} \widetilde{\mathbf{G}}^{l}-2 \sum_{l=1}^{L} \sum_{k=1}^{K}\left(\widetilde{\mathbf{G}}^{l}\right)^{T} E^{k}\left(E^{k}\right)^{T} \widetilde{\mathbf{G}}^{l} \\
& +\sum_{l=1}^{L} \sum_{k_{1}=1}^{K} \sum_{k_{2}=1}^{K}\left(\widetilde{\mathbf{G}}^{l}\right)^{T} E^{k_{1}}\left(E^{k_{1}}\right)^{T} E^{k_{2}}\left(E^{k_{2}}\right)^{T} \widetilde{\mathbf{G}}^{l} .
\end{aligned}
$$

The usage of the orthonormal property of $E^{k}$, with $k=1, \ldots, K$, yields

$$
\mathfrak{L}=\sum_{l=1}^{L}\left(\left(\widetilde{\mathbf{G}}^{l}\right)^{T} \widetilde{\mathbf{G}}^{l}-\sum_{k=1}^{K}\left(\widetilde{\mathbf{G}}^{l}\right)^{T} E^{k}\left(E^{k}\right)^{T} \widetilde{\mathbf{G}}^{l}\right) .
$$

Since the quantities $\left(\widetilde{\mathbf{G}}^{l}\right)^{T} E^{k}$ and $\left(E^{k}\right)^{T} \widetilde{\mathbf{G}}^{l}$ are scalars, their order of multiplication is reversed to yield

$$
\mathfrak{L}=\sum_{l=1}^{L}\left(\left(\widetilde{\mathbf{G}}^{l}\right)^{T} \widetilde{\mathbf{G}}^{l}-\sum_{k=1}^{K}\left(E^{k}\right)^{T} \widetilde{\mathbf{G}}^{l}\left(\widetilde{\mathbf{G}}^{l}\right)^{T} E^{k}\right) .
$$

A Lagrange function $\tilde{\mathfrak{L}}$ is constructed by introducing new variables $\lambda_{k}, i=1,2, \ldots, K$, to incorporate the unit length constraints on the energy vector and is given by

$$
\tilde{\mathfrak{L}}=\mathfrak{L}+\sum_{k=1}^{K} \lambda_{k}\left(\left(E^{k}\right)^{T} E^{k}-1\right) .
$$

Taking the matrix derivatives of $\tilde{\mathfrak{L}}$ with respect to the energy vectors and setting them equal to zero yield

$$
\begin{aligned}
\frac{\partial \tilde{\mathfrak{L}}}{\partial E^{k}}=-2\left(E^{k}\right)^{T}\left(\sum_{l=1}^{L}\left(\widetilde{\mathbf{G}}^{l}\right)\left(\widetilde{\mathbf{G}}^{l}\right)^{T}\right)+2 \lambda_{k}\left(E^{k}\right)^{T} & =0 \\
\Rightarrow\left(\sum_{l=1}^{L}\left(\widetilde{\mathbf{G}}^{l}\right)\left(\widetilde{\mathbf{G}}^{l}\right)^{T}\right) E^{k} & =\lambda_{k} E^{k} .
\end{aligned}
$$

The energy vectors are estimated as the eigenvectors of the matrix $\left(\sum_{l=1}^{L}\left(\widetilde{\mathbf{G}}^{l}\right)\left(\widetilde{\mathbf{G}}^{l}\right)^{T}\right)$. The symmetry property of the matrix implies that the energy vectors are orthogonal to each other. 


\section{REFERENCES}

[1] X. Wang, S. Wang, and D. Bi, "Distributed visual-target-surveillance system in wireless sensor networks," IEEE Trans. Syst., Man, Cybern. B, Cybern., vol. 39, no. 5, pp. 1134-1146, Oct. 2009.

[2] S. Phoha, T. La Porta, and C. Griffin, Sensor Network Operations. Piscataway, NJ: Wiley-IEEE Press, May 2006.

[3] S. Ferrari, R. Fierro, B. Perteet, C. Cai, and K. Baumgartner, "A geometric optimization approach to detecting and interfacing dynamic targets using a mobile sensor network," SIAM J. Control Optim., vol. 48, no. 1, pp. 292 $320,2009$.

[4] H. M. Shertukde and Y. Bar-Shalom, "Detection and estimation for multiple targets with two omnidirectional sensors in the presence of false measurements," IEEE Trans. Acoust., Speech, Signal Process., vol. 38, no. 5, pp. 749-763, May 1990.

[5] T. Wettergren, "Performance of search via track-before-detect for distributed sensor networks," IEEE Trans. Aerosp. Electron. Syst., vol. 44, no. 1, pp. 314-325, Jan. 2008.

[6] T. Wettergren and R. Costa, "Optimal placement of distributed sensors against moving targets," ACM Trans. Sens. Netw., vol. 5, no. 3, pp. 1-25, May 2009.

[7] L. D. Stone, Theory of Optimal Search. Mathematics in Science and Engineering, vol. 118. New York: Academic, 1975.

[8] S. Meguerdichian, F. Koushanfar, G. Qu, and M. Potkonjak, "Exposure in wireless ad-hoc sensor networks," in Proc. 7th Annu. Int. Conf. MobiCom, 2001, pp. 139-150.

[9] S. S. Ram, D. Manjunath, S. K. Iyer, and D. Yogeshwaran, "On the path coverage properties of random sensor networks," IEEE Trans. Mobile Comput., vol. 6, no. 5, pp. 494-506, May 2007.

[10] T. Clouqueur, V. Phipatanasuphorn, P. Ramanathan, and K. K. Saluja, "Sensor deployment strategy for target detection," in Proc. 1st ACM Int. Workshop WSNA, 2002, pp. 42-48.

[11] K. Baumgartner and S. Ferrari, "A geometric transversal approach to analyzing track coverage in sensor networks," IEEE Trans. Comput., vol. 57, no. 8, pp. 1113-1128, Aug. 2008

[12] K. Baumgartner, S. Ferrari, and A. Rao, "Optimal control of a mobile sensor network for cooperative target detection," IEEE J. Ocean. Eng., vol. 34, no. 4, pp. 678-697, Oct. 2009.

[13] K. Huang, Introduction to Statistical Physics. New York: Taylor \& Francis, 2001

[14] S. Gupta and A. Ray, "Statistical mechanics of complex systems for pattern identification," J. Stat. Phys., vol. 134, no. 2, pp. 337-364, Jan. 2009.

[15] R. Albert and A.-L. Barabasi, "Statistical mechanics of complex networks," Rev. Mod. Phys., vol. 74, no. 1, pp. 47-97, Jan. 2002.

[16] H. Van Dyke Parunak and S. Brueckner, "Entropy and self-organization in multi-agent systems," in Proc. 5th Int. Conf. Auton. Agents (AGENTS), 2001, pp. 124-130.

[17] S. Gupta, A. Ray, and S. Phoha, "Generalized Ising model for dynamic adaptation in autonomous systems," Eur. Phys. Lett. (EPL), vol. 87, no. 1, p. 10009 , Jul. 2009

[18] L. Barnes, M. Fields, and K. Valavanis, "Swarm formation control utilizing elliptical surfaces and limiting functions," IEEE Trans. Syst., Man, Cybern. B, Cybern., vol. 39, no. 6, pp. 1434-1445, Dec. 2009.

[19] I. Chattopadhyay and A. Ray, "Supervised self-organization of homogeneous swarms using projections of Markov chains," IEEE Trans. Syst., Man, Cybern. B, Cybern., vol. 39, no. 6, pp. 1505-1515, Dec. 2009.

[20] T. Hogg, Applications of Statistical Mechanics to Combinatorial Search Problems, vol. 2. Singapore: World Scientific, 1995, pp. 357-406.

[21] E. Aarts and J. Korst, Simulated Annealing and Boltzmann Machines: A Stochastic Approach to Combinatorial Optimization and Neural Computing. New York: Wiley, 1989.

[22] Y. Bar-Shalom, X. R. Li, and T. Kirubarajan, Estimation With Applications to Tracking and Navigation. New York: Wiley, 2001.

[23] R. Steuer, Multiple Criteria Optimization: Theory, Computations, and Applications. New York: Wiley, 1986.

[24] T. Wettergren, "The genetic-based normal boundary intersection (GANBI) method: An efficient approach to Pareto multiobjective optimization for engineering design," Naval Undersea Warfare Center Div., Newport, RI, NUWC Tech. Rep. 11, 741, 2006.

[25] C. Belta and V. Kumar, "Abstraction and control for groups of robots," IEEE Trans. Robot., vol. 20, no. 5, pp. 865-875, Oct. 2004.

[26] K. Fukunaga, Statistical Pattern Recognition, 2nd ed. Boston, MA: Academic, 1990.

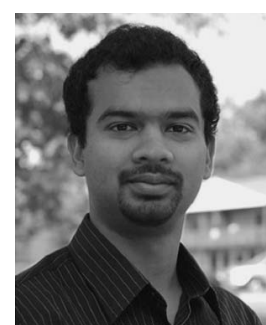

Kushal Mukherjee (S'08) received the B.S. degree in mechanical engineering from the Indian Institute of Technology, Roorkee, India, in 2006, and the M.S. degrees in mechanical and electrical engineering from Pennsylvania State University, University Park, in 2009 , where he is currently working toward the $\mathrm{Ph} . \mathrm{D}$. degree in mechanical engineering.

His areas of interest include symbolic dynamics, sensor networks, and multiagent systems.

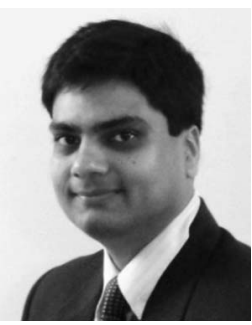

Shalabh Gupta (M'04) received the M.S. degrees in mechanical and electrical engineering and the Ph.D. degree in mechanical engineering in 2006 from Pennsylvania State University (Penn State), University Park

$\mathrm{He}$ is currently a Research Associate in the Department of Mechanical Engineering, Penn State. His research interests include the science of autonomy, swarm robotics, intelligent systems, machine learning, network science, and fault detection and isolation in complex systems. His research efforts have been instrumental in opening new fields of data understanding and pattern discovery by interfacing multidisciplinary concepts derived from statistical mechanics, symbolic dynamics, and languages and automata theory.

Dr. Gupta is a member of the American Society of Mechanical Engineers.

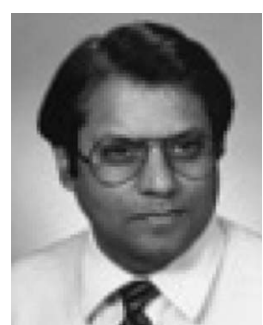

Asok Ray (SM'83-F'02) received the Ph.D. degree in mechanical engineering from Northeastern University, Boston, MA, and graduate degrees in electrical engineering, mathematics, and computer science.

He joined Pennsylvania State University (Penn State), University Park, in July 1985, where he is currently a Distinguished Professor of Mechanical Engineering. Prior to joining Penn State, he also held research and academic positions at Massachusetts Institute of Technology, Cambridge, and CarnegieMellon University, Pittsburgh, PA, as well as research and management positions at GTE Strategic Systems Division, Charles Stark Draper Laboratory, and MITRE Corporation. He was a Senior Research Fellow at the NASA Glenn Research Center under a National Academy of Sciences award. His research experience and interests include control and optimization of continuously varying and discrete-event dynamical systems, intelligent instrumentation for real-time distributed systems, and modeling and analysis of complex dynamical systems from thermodynamic perspectives in both deterministic and stochastic settings, as applied to robotics, undersea autonomous vehicles, aeronautics and astronautics, and fossil-fueled and nuclear power plants. He has authored or coauthored over 450 research publications, including about 250 scholarly articles in refereed journals, such as transactions of IEEE, ASME, and AIAA, and research monographs.

Dr. Ray is a Fellow of the American Society of Mechanical Engineers and of the World Innovative Foundation.

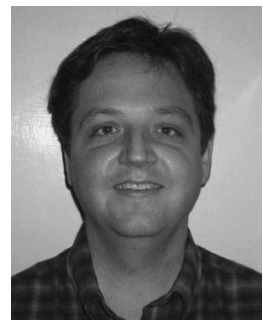

Thomas A. Wettergren (A'95-M'98-SM'06) received the B.S. degree in electrical engineering and the Ph.D. degree in applied mathematics from Rensselaer Polytechnic Institute, Troy, NY.

He joined the Naval Undersea Warfare Center, Newport, RI, in 1995, where he has served as a Research Scientist in the torpedo systems, sonar systems, and undersea combat systems departments. He currently serves as the U.S. Navy Senior Technologist for Operational and Information Science as well as a Senior Research Scientist at the Newport laboratory. His personal research interests are in mathematical modeling, analysis, optimization, and control of undersea sensing systems.

Dr. Wettergren is a member of the Society for Industrial and Applied Mathematics. 